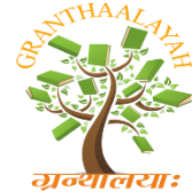

INTERNATIONAL JOURNAL OF RESEARCH GRANTHAALAYAH A knowledge Repository

RAST - 17

\title{
A MATHEMATICAL APPROACH TO STUDY THE EFFECT OF POLLUTANTS/TOXICANTS IN AQUATIC ENVIRONMENT
}

\author{
Anita Chaturvedi ${ }^{* 1}$, Kokila Ramesh ${ }^{2}$, Vatsala G A ${ }^{3}$ \\ ${ }^{* 1}$ Professor, Department of Basic Sciences, School of Engineering and Technology, Jain \\ University, India \\ ${ }^{2}$ Assistant Professor, Department of Basic Sciences, School of Engineering and Technology, \\ Jain University, India \\ ${ }^{3}$ Associate Professor, Department of Mathematics, Dayanand Sagar Academy of Technology and \\ Management, India \\ DOI: https://doi.org/10.29121/granthaalayah.v5.i4RAST.2017.3299
}

\begin{abstract}
Acid lowers the $\mathrm{pH}$ levels in water bodies below what is required for survival of aquatic life and increases the toxicity of metals. For this effect, a mathematical model has been proposed using a system of non-linear ordinary differential equations with four state variables. The dependent variables are amount of acid and metal in water, density of favorable resources (phytoplankton), density of fish population and nutrient concentration under the assumption that the amount of metal present in water is less than the amount of acid present in water. Conditions for local stability and feasible equilibrium points have been determined. Nonlinear stability analysis of the non-trivial equilibrium points has been discussed and it was found that system of the differential equations show more feasible results if the crowding effect is incorporated for fish population. Further it was also observed that, nutrients play important role for the growth and survival of the species. Conditions for the existence of the equilibrium points have been drawn and the criteria for the survival or the extinction of the species have been obtained using numerical simulation. Stability of the system is explained analytically as well as graphically.
\end{abstract}

Keywords: Nutrient Cycle; Crowding Effect; Lyapunov's Function; Feasible Equilibria; State Variables.

Cite This Article: Anita Chaturvedi, Kokila Ramesh, and Vatsala G A. (2017). "A MATHEMATICAL APPROACH TO STUDY THE EFFECT OF POLLUTANTS/TOXICANTS IN AQUATIC ENVIRONMENT." International Journal of Research - Granthaalayah, 5(4) RAST, 33-38. 10.29121/granthaalayah.v5.i4RAST.2017.3299. 


\section{Introduction}

Acid precipitation, either falling directly on aquatic bodies or what gets run off the forests, fields or roads may cause increased levels of acid in water bodies. The acid accumulation over a period of time lowers the overall $\mathrm{pH}$. The aquatic plants and animals require $\mathrm{pH}$ of around 4.8. Any lower value makes the conditions hostile for survival. Metal are introduced in aquatic life through weathering of soils, rocks from volcanic eruption and mining. The most common source in fresh water bodies is mining. Metals are highly soluble in acid solution. The drainage process from ores releases heavy metals and the acid solution is dispersed in the groundwater with high levels of metal. When the $\mathrm{pH}$ levels of waterfall decreases, metal solubility increases causing toxicity of metals to increase. However, some metals like manganese, iron, copper and zinc are essential micronutrients in the right concentrations but in excess become toxic. Also, it is known that fish species vary widely in the rates at which they excrete nutrients like nitrogen and phosphorus. Hence altering communities may have an effect on nutrient cycling. Fish store a large portion of ecosystem nutrients in their tissues, transporting them farther and faster than other aquatic animals. They also excrete dietary nutrients in dissolved forms making them available to producers. Nutrient cycling provides a quantitative basis linking population and eco functioning. Taking these factors a mathematical model has been proposed incorporating crowding effect of fish. A system of non-linear differential equations including state variablesnutrients, favorable resources, fish population, acid and metal concentrations have been analyzed both mathematically and by numerical simulation. The feasible equilibria and Lyapunov's stability conditions have been determined.

\section{Mathematical Model}

In the model we consider, $S(t)$ is the concentration of nutrient in the water, $P(t)$ is the density of favorable resources, $F(t)$ is the density of fish population, $T(t)$ is the concentration of acid in water and metal in water where $(0<q<1)$. Mathematical model of the governing system having the above mentioned four state variables is structured as follows:

$\frac{d S}{d t}=S_{0}-a S-g S P-\alpha\left(T_{1}+q C_{m}\right) S+K c P+K b F+K_{1} F^{2}$

$\frac{d P}{d t}=g S P-c P-f F P$

$\frac{d F}{d t}=f P F-b F-K F^{2}$

$\frac{d\left(T_{1}+q C_{m}\right)}{d t}=Q_{0}-\alpha\left(T_{1}+q C_{m}\right)-\alpha_{1}\left(T_{1}+q C_{m}\right) S$

With the initial conditions:

$S(0)=S_{10}>0, P(0)=P_{10}>0, F(0)=F_{10}>0, T(0)=T_{10}>0$

Where,

$S_{0}, a, b, c, f, g, \alpha_{1}, \alpha, Q_{0}, K, K_{1}$ are positive constants. Now Considering $T=\left(T_{1}+q C_{m}\right)$

Then the System reduces to-

$$
\frac{d S}{d t}=S_{0}-a S-g S P-T S+K c P+K b F+K_{1} F^{2}
$$


$\frac{d P}{d t}=g S P-c P-f F P$

$\frac{d F}{d t}=f P F-b F-K F^{2}$

$\frac{d T}{d t}=Q_{0}-\alpha T-\alpha_{1} T S$

With the initial conditions:

$S(0)=S_{10}>0, P(0)=P_{10}>0, F(0)=F_{10}>0, T(0)=T_{10}>0$

Where, $Q_{0}$ is the constant input rate of pollutant in the water, $S_{0}$ is the constant nutrient in the water, $a$ is the nutrient leaching rate, $g$ is the rate of consumption of nutrient by resource, $\alpha_{1}$ is the depletion rate of nutrient due to pollution concentration in the water, $f$ is the specific rate of predation of fish on resource, $c$ is the natural death rates of resource, $b$ is the natural death rates of fish population, $\alpha$ is the natural washout rate of pollutant from the water and $K(0<K<1)$ determines a proportionate amount of resource and fish population that is being recycled back to the nutrient pool after death, $K_{1}$ is the crowding effect of fish in water.

$S_{0}, a, b, c, f, g, \alpha_{1}, \alpha, Q_{0}, K, K_{1}$ are positive constants

\section{Boundedness and Dynamical Behaviour}

The boundedness of solutions of the model (1A) - (4A) is given by the following lemma

Lemma 1: All the solutions of the model (1A) - (4A) will lie in the region:-

$\mathrm{A}=\left\{(S, P, F, T) \in R_{+}^{4} \quad 0 \leq \frac{S_{0}+Q_{0}}{\theta_{1}} \leq S+P+F+T ; 0 \leq S+P+F \leq \frac{S_{0}}{\theta} ; 0 \leq T \leq \frac{Q_{0}}{\alpha}\right\}$ as $t \rightarrow \infty$ for all positive initial values $\left\{S_{10}, P_{10}, F_{10}, T_{10}\right\} \in R_{+}^{4}$ where $\theta=\min [(1-K) c,(1-$ $K) b]$ and $\theta_{1}=\max \left[\left(a+2 \alpha_{1} \frac{Q_{0}}{\alpha}\right), c, b, \alpha\right]$

$\underline{\text { Proof: }}$ Let us consider the following function $W(t)=S(t)+P(t)+F(t)$

From equation (1A) - (3A) and if $\theta=\min [(1-K) c,(1-K) b]$ then we obtain $W(t) \leq S_{0}-$ $\theta W$

Then by usual comparison we get the following result as $t \rightarrow \infty$

$W(t) \leq \frac{S_{0}}{\theta} \Rightarrow S(t)+P(t)+F(t) \leq \frac{S_{0}}{\theta}$ and $T \leq \frac{Q_{0}}{\alpha}$

Again, we consider the following function:

$W_{1}(t)=W(t)+T(t)$

From equations (1A) - (4A), and if $\left.\theta_{1}=\max \left[\left(a+2 \alpha_{1} \frac{Q_{0}}{\alpha}\right), c, b, \alpha\right)\right]$

Then we obtain the following expression

$W_{1}(t) \geq S_{0}+Q_{0}-\theta_{1} W_{1}$

Then by usual comparison, we get $t \rightarrow \infty$

$W_{1}(t) \geq \frac{S_{0}+Q_{0}}{\theta_{1}} \Rightarrow S(t)+P(t)+F(t)+T(t) \geq \frac{S_{0}+Q_{0}}{\theta_{1}}$

From equation (4A) we have $\alpha T(t) \leq Q_{0} \Rightarrow T(t) \leq \frac{Q_{0}}{\alpha}$. Hence the proof of the lemma 


\section{Equilibrium}

Now we will discuss the uniform equilibrium points and interior equilibrium point. The model has the following set of non-negative equilibrium points.

i) The first equilibrium point $E_{1}:(\tilde{S} \neq 0, \tilde{P}=0, \tilde{F}=0, \tilde{T} \neq 0)$

$$
\begin{aligned}
& \text { where } \tilde{S}=\frac{S_{0}}{a+\alpha T} \text { and } \tilde{T} \text { is given by } \tilde{T}=\frac{-g_{2}+\sqrt{g_{2}^{2}-4 g_{1} g_{3}}}{2 g_{1}}>0 \\
& \text { here } g_{1}=\alpha, g_{2}=a-Q_{0} \alpha^{2}, g_{3}=-\left(Q_{0} a \alpha+\alpha_{1} S_{0}\right) \\
& \text { If } \sqrt{g_{2}^{2}-4 g_{1} g_{3}}>g_{2},-4 g_{1} g_{3}>0
\end{aligned}
$$

ii) The second equilibrium $E_{2}:(\bar{S} \neq 0, \bar{P} \neq 0, \bar{F}=0, \bar{T} \neq 0)$

$$
\begin{aligned}
& \text { where, } \bar{S}=\frac{c}{g}, \bar{T}=\frac{Q_{0}}{g \alpha+\alpha_{1} c} \text { and } \bar{P}=\frac{S_{0} g\left(g \alpha+\alpha_{1} c\right)-\left[a c\left(g \alpha+\alpha_{1} c\right)+\alpha Q_{0} g^{2}\right]}{(1-K) c g\left(g \alpha+\alpha_{1} c\right)} \\
& \text { If } S_{0} g\left(g \alpha+\alpha_{1} c\right)>a c\left(g \alpha+\alpha_{1} c\right)+\alpha Q_{0} g^{2}
\end{aligned}
$$

iii) Interior equilibrium $E_{3}:\left(S^{*} \neq 0, P^{*} \neq 0, F^{*} \neq 0, T^{*} \neq 0\right)$

$$
\text { where, } P^{*}=\frac{b+k F^{*}}{f}, F^{*}=\frac{g S^{*}-c}{f}, T^{*}=\frac{Q_{0}}{\alpha+\alpha_{1} S^{*}} \text { and } S^{*}=\frac{S_{0}+k c P^{*}+K b F^{*}+K_{1} F^{2}}{a+g P^{*}+\alpha T^{*}}
$$

$$
S^{*}=\frac{-h_{2}+\sqrt{h_{2}^{2}-4 h_{1} h_{3}}}{2 h_{1}}>0
$$

where, $h_{1}=a f \alpha_{1}+g b \alpha_{1}-K b g \alpha_{1}$

$$
\begin{aligned}
& \quad h_{2}=a f \alpha+g b \alpha+\alpha Q_{0} f-k b g \alpha-\alpha_{1} S_{0} f-K c b \alpha_{1}+K b c \alpha \\
& h_{3}=-S_{0} f \alpha \\
& \text { If } \sqrt{h_{2}^{2}-4 h_{1} h_{3}}>h_{2} \Rightarrow-4 h_{1} h_{3}>0
\end{aligned}
$$

Now we will discuss the local behavior of the dynamical system (1A)-(4A) around the equilibrium

$$
E_{i}(i=1,2,3)
$$

\section{Numerical Example}

Consider the following set of parameters:

$S_{0}=1, Q_{0}=1, a=0.1, b=0.1, c=0.1, K=0.01, f=0.09, g=0.08, \alpha_{1}=0.35, \alpha=0.3$, $K_{1}=0.005, \theta=0.099, \theta_{1}=2.43333$

The region of attraction is:

$(S, P, F, T) \in R_{+}^{4} \quad 0 \leq 0.821929067 \leq S+P+F+T ; 0 \leq S+P+F \leq 10.101010 ; 0 \leq T \leq$ 3.333 ; and the interior equilibrium of the model is $E_{3}=(2.00787,1.1860,0.6744711,0.996936)$ 
[Chaturvedi et. al., Vol.5 (Iss.4: RAST), April, 2017]

ICV (Index Copernicus Value) 2015: 71.21

Recent Advances in Science \& Technology

The time series plot and the phase space are shown in Figure 1 and Figure 2.
ISSN- 2350-0530(O), ISSN- 2394-3629(P) IF: 4.321 (CosmosImpactFactor), 2.532 (I2OR)

InfoBase Index IBI Factor 3.86

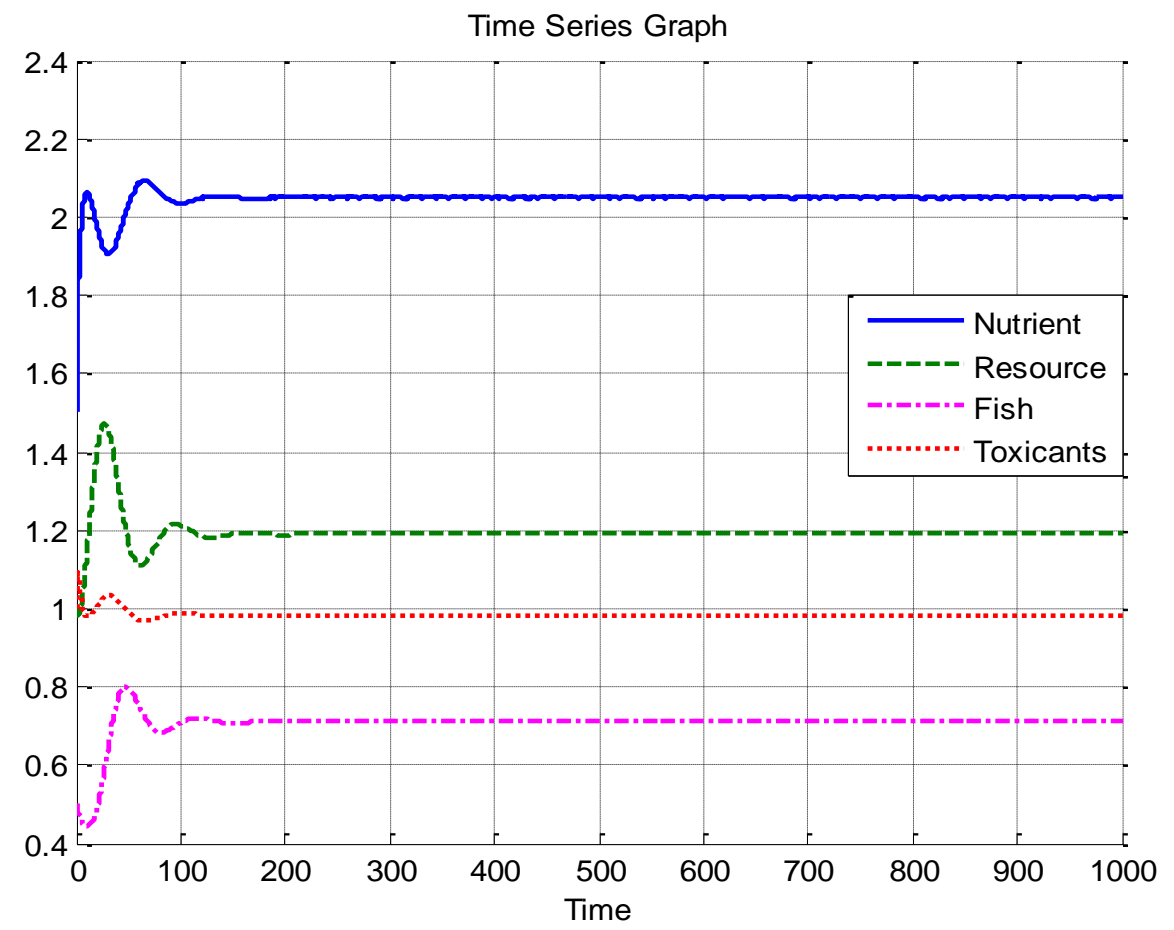

Figure 1: The variation of Nutrient, Resource, Fish and the Toxicants with time
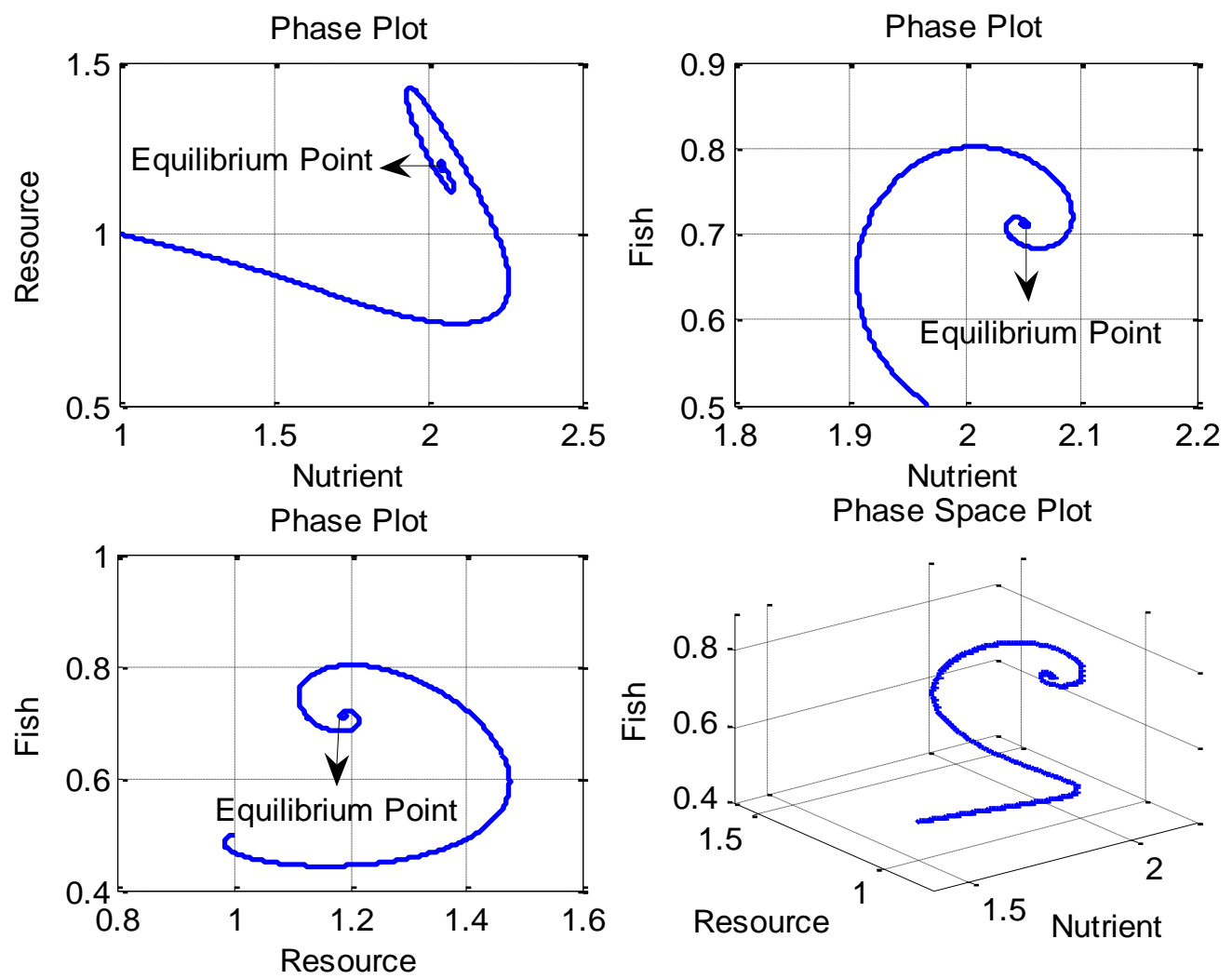

Figure 2: The interaction between the Nutrient and the Resource, Nutrient and the Fish, Resource and the Fish and between Nutrient, Resource and Fish 


\section{Conclusions}

From the above analysis it can be concluded that toxicity in the nutrient pool causes increase in equilibrium level of resource and fish populations. The interior equilibrium exists only when the non-living equilibrium and fish extinct equilibrium are unstable. The non-living equilibriums are stable, if the equilibrium level of concentration of nutrient in water is less than the fraction of natural death rate of resource biomass to the rate of consumption of nutrient by the resource population. The fish extinct equilibrium is stable, if the equilibrium level of resource population in water is less than fraction of natural death rate of fish population to the specific rate of predation of fish on resource population. Interior equilibrium is locally as well as non-linearly asymptotically stable under certain conditions .The interior equilibrium is sensitive to specific rate of predation of fish on resource population. In the numerical example, for a set of parameters, interior equilibrium points for all the four state variables are calculated. It has been observed that even in the numerical simulation, the variables have reached the equilibrium points. Hence the calculated and the simulated values match with each other. Also the interactions between the variables have been studied in the form of phase plots.

\section{References}

[1] Asha Bharathi A.T, Anita Chaturvedi, Radha Gupta and Kokila Ramesh (2015):COMBINED EFFECTS OF ACID AND METAL ON THE SURVIVAL OF RESOURCE BASED POPULATION INCORPORATING NUTRIENT RECYCLING: A MATHEMATICAL MODEL: International Journal of Applied :Engineering Research ISSN 0973-4562 Volume 10, Number 6 pp. 14969-14980.

[2] Chaturvedi, Misra (2011): COMBINED EFFECTS OF ACID AND METAL ON RESOURCE BASED INTERACTING AQUATIC SPECIES SYSTEM. JIAM Vol.33,No1.

[3] Swati Khare, (2010): MODELLING EFFECTS OF POLLUTANTS ON INTERACTING SPECIES SYSTEM WITH NUTRIENT CYCLING. Nonlinear Analysis: Hybrid Systems. 4 (2010), pp. 496 - 502

[4] Swati Khare, O.P. Misra and Preety Kalra (2009): EFFECT OF TOXICANT ON EXISTENCE OF SINGLE SPECIES SYSs Proceedings of Mathematical Society, B.H.U., Vol 21.

[5] Chattopadhyay J., (2005): PLANKTON BLOOM: IMPACT TOXIN PRODUCING PHYTOPLANKTON, WORKSHOP ON "NONLINEAR DYNAMICAL MODELS AND THEIR BEHAVIOR", 42-64, 11-13 March, Dept. of Math., IIT Roorkee (U.P).

[6] O.P.Misra, S.K.S.Rathor and Y.N.Meitei (2003): A MATHEMATICAL MODEL FOR THE SURVIVAL OR EXTINCTION OF RESOURCE DEPENDENT SPECIES UNDER POLLUTION STRESS AND INDUSTRIALIZATION PRESSURE, Proceeding of Mathematical Society, B.H.U., Vol 19.

\footnotetext{
*Corresponding author.

E-mail address: acvedi_05@yahoo.com
} 